\title{
Research Article \\ Stability Problem of Ulam for Euler-Lagrange Quadratic Mappings
}

\author{
Hark-Mahn Kim, John Michael Rassias, and Young-Sun Cho \\ Received 26 May 2007; Revised 9 August 2007; Accepted 9 November 2007 \\ Recommended by Ondrej Dosly
}

We solve the generalized Hyers-Ulam stability problem for multidimensional EulerLagrange quadratic mappings which extend the original Euler-Lagrange quadratic mappings.

Copyright (c) 2007 Hark-Mahn Kim et al. This is an open access article distributed under the Creative Commons Attribution License, which permits unrestricted use, distribution, and reproduction in any medium, provided the original work is properly cited.

\section{Introduction}

In 1940, Ulam [1] proposed, at the University of Wisconsin, the following problem: "give conditions in order for a linear mapping near an approximately linear mapping to exist." In 1968, Ulam proposed the general Ulam stability problem: "when is it true that by changing a little the hypotheses of a theorem one can still assert that the thesis of the theorem remains true or approximately true?" The concept of stability for a functional equation arises when we replace the functional equation by an inequality which acts as a perturbation of the equation. Thus the stability question of functional equations is "how do the solutions of the inequality differ from those of the given functional equation?" If the answer is affirmative, we would say that the equation is stable. In 1978, Gruber [2] remarked that Ulam problem is of particular interest in probability theory and in the case of functional equations of different types. We wish to note that stability properties of different functional equations can have applications to unrelated fields. For instance, Zhou [3] used a stability property of the functional equation $f(x-y)+f(x+y)=2 f(x)$ to prove a conjecture of $Z$. Ditzian about the relationship between the smoothness of a mapping and the degree of its approximation by the associated Bernstein polynomials.

Above all, Ulam problem for $\varepsilon$-additive mappings $f: E_{1} \rightarrow E_{2}$ between Banach spaces, that is, $\|f(x+y)-f(x)-f(y)\| \leq \varepsilon$ for all $x, y \in E_{1}$, was solved by Hyers [4] and then generalized by Th. M. Rassias [5] and Găvruţa [6] who permitted the Cauchy difference 
to become unbounded. The stability problems of several functional equations have been extensively investigated by a number of authors and there are many interesting results concerning this problem. A large list of references can be found, for example, in [7-9] and references therein.

We note that J. M. Rassias introduced the Euler-Lagrange quadratic mappings, motivated from the following pertinent algebraic equation

$$
\left|a_{1} x_{1}+a_{2} x_{2}\right|^{2}+\left|a_{2} x_{1}-a_{1} x_{2}\right|^{2}=\left(a_{1}^{2}+a_{2}^{2}\right)\left[\left|x_{1}\right|^{2}+\left|x_{2}\right|^{2}\right] .
$$

Thus the second author of this paper introduced and investigated the stability problem of Ulam for the relative Euler-Lagrange functional equation

$$
f\left(a_{1} x_{1}+a_{2} x_{2}\right)+f\left(a_{2} x_{1}-a_{1} x_{2}\right)=\left(a_{1}^{2}+a_{2}^{2}\right)\left[f\left(x_{1}\right)+f\left(x_{2}\right)\right]
$$

in the publications [10-12]. Analogous quadratic mappings were introduced and investigated through J. M. Rassias publications [13-15]. Therefore, these mappings could be named Euler-Lagrange mappings and the corresponding Euler-Lagrange equations might be called Euler-Lagrange equations. Before 1992, these mappings and equations were not known at all in functional equations and inequalities. However, a completely different kind of Euler-Lagrange partial differential equations is known in calculus of variations. Already, some mathematicians have employed these Euler-Lagrange mappings [16-22].

In addition, J. M. Rassias [23] generalized the above functional equation (1.2) as follows. Let $X$ and $Y$ be real linear spaces. Then a mapping $Q: X \rightarrow Y$ is called quadratic with respect to $a$ if the functional equation

$$
Q\left(\sum_{i=1}^{n} a_{i} x_{i}\right)+\sum_{1 \leq i<j \leq n} Q\left(a_{j} x_{i}-a_{i} x_{j}\right)=\left(\sum_{i=1}^{n} a_{i}^{2}\right) \sum_{i=1}^{n} Q\left(x_{i}\right)
$$

holds for all vector $\left(x_{1}, \ldots, x_{n}\right) \in X^{n}$, where $a:=\left(a_{1}, \ldots, a_{n}\right) \in \mathbb{R}^{n}$ of nonzero reals, and $n \geq$ 2 is arbitrary, but fixed, such that $0<m:=\sum_{i=1}^{n} a_{i}^{2} \neq\left[1+\left(\begin{array}{c}n \\ 2\end{array}\right)\right] / n$. In this case, a mapping $\bar{Q}^{a}: X^{n} \rightarrow Y$ defined by

$$
\bar{Q}^{a}\left(x_{1}, \ldots, x_{n}\right):=\frac{\sum_{i=1}^{n} Q\left(a_{i} x_{i}\right)}{\sum_{i=1}^{n} a_{i}^{2}}
$$

is called the square of the quadratic weighted mean of $Q$ with respect to $a$.

For every $x \in \mathbb{R}$, set $Q(x)=x^{2}$. Then the mapping $\bar{Q}^{a}: \mathbb{R}^{n} \rightarrow \mathbb{R}$ is quadratic such that $\bar{Q}^{a}(x, \ldots, x)=x^{2}$. Denoting by $\sqrt{\overline{x_{w}^{2}}}$ the quadratic weighted mean, we note that the abovementioned mapping $\bar{Q}^{a}$ is an analogous case to the square of the quadratic weighted mean employed in mathematical statistics: $\overline{x_{w}^{2}}=\sum_{i=1}^{n} w_{i} x_{i}^{2} / \sum_{i=1}^{n} w_{i}$ with weights $w_{i}=a_{i}^{2}$, data $x_{i}$, and $Q\left(a_{i} x_{i}\right)=\left(a_{i} x_{i}\right)^{2}$ for $i=1, \ldots, n$, where $n \geq 2$ is arbitrary, but fixed.

In this paper, using the iterative methods and ideas inspired by $[6,23]$, we are going to investigate the generalized Hyers-Ulam stability problem for the quadratic functional equation of Euler-Lagrange (1.3). 


\section{Stability of (1.3) in quasi-Banach spaces}

We will investigate under what conditions it is then possible to find a true quadratic mapping of Euler-Lagrange near an approximate Euler-Lagrange quadratic mapping with small error.

We recall some basic facts concerning quasi-Banach spaces and some preliminary results.

Definition 2.1 (see $[24,25]$ ). Let $X$ be a linear space. A quasinorm $\|\cdot\|$ is a real-valued function on $X$ satisfying the following:

(1) $\|x\| \geq 0$ for all $x \in X$ and $\|x\|=0$ if and only if $x=0$;

(2) $\|\lambda x\|=|\lambda| \cdot\|x\|$ for all $\lambda \in \mathbb{R}$ and all $x \in X$;

(3) there is a constant $K$ such that $\|x+y\| \leq K(\|x\|+\|y\|)$ for all $x, y \in X$.

The smallest possible $K$ is called the modulus of concavity of $\|\cdot\|$. The pair $(X,\|\cdot\|)$ is called a quasinormed space if $\|\cdot\|$ is a quasinorm on $X$. A quasi-Banach space is a complete quasinormed space.

A quasinorm $\|\cdot\|$ is called a $p$-norm $(0<p \leq 1)$ if

$$
\|x+y\|^{p} \leq\|x\|^{p}+\|y\|^{p}
$$

for all $x, y \in X$. In this case, a quasi-Banach space is called a $p$-Banach space.

Clearly, $p$-norms are continuous, and in fact, if $\|\cdot\|$ is a $p$-norm on $X$, then the formula $d(x, y):=\|x-y\|^{p}$ defines a translation invariant metric for $X$, and $\|\cdot\|^{p}$ is a $p$ homogeneous $F$-norm. The Aoki-Rolewicz theorem [24, 25] guarantees that each quasinorm is equivalent to some $p$-norm for some $0<p \leq 1$. In this section, we are going to prove the generalized Ulam stability of mappings satisfying approximately (1.3) in quasiBanach spaces, and in $p$-Banach spaces, respectively. Let $X$ be a quasinormed space and $Y$ a quasi-Banach space with the modulus of concavity $K \geq 1$ of $\|\cdot\|$.

Given a mapping $f: X \rightarrow Y$, we define a difference operator $D_{a} f: X^{n} \rightarrow Y$ for notational convenience as

$$
D_{a} f\left(x_{1}, \ldots, x_{n}\right):=f\left(\sum_{i=1}^{n} a_{i} x_{i}\right)+\sum_{1 \leq i<j \leq n} f\left(a_{j} x_{i}-a_{i} x_{j}\right)-\left(\sum_{i=1}^{n} a_{i}^{2}\right) \sum_{i=1}^{n} f\left(x_{i}\right),
$$

which is called the approximate remainder of the functional equation (1.3) and acts as a perturbation of the equation, where $a:=\left(a_{1}, \ldots, a_{n}\right) \in \mathbb{R}^{n}$ of nonzero reals, and $n \geq 2$ is arbitrary, but fixed, such that $0<m:=\sum_{i=1}^{n} a_{i}^{2} \notin\left\{\left[1+\left(\begin{array}{c}n \\ 2\end{array}\right)\right] / n, \sqrt{K}\right\}$.

Lemma 2.2 [23]. Let $Q: X \rightarrow Y$ be a Euler-Lagrange quadratic mapping satisfying (1.3). Then $Q$ satisfies the equation

$$
Q\left(m^{p} x\right)=m^{2 p} Q(x)
$$

for all $x \in X$ and $p \in \mathbb{N}$, where $0<m:=\sum_{i=1}^{n} a_{i}^{2} \neq\left[1+\left(\begin{array}{c}n \\ 2\end{array}\right)\right] / n(\geq 1)$. 
4 Journal of Inequalities and Applications

Theorem 2.3. Assume that there exists a mapping $\varphi: X^{n} \rightarrow[0, \infty)$ for which a mapping $f: X \rightarrow Y$ satisfies

$$
\left\|D_{a} f\left(x_{1}, \ldots, x_{n}\right)\right\| \leq \varphi\left(x_{1}, \ldots, x_{n}\right)
$$

and the series

$$
\Phi\left(x_{1}, \ldots, x_{n}\right):=\sum_{i=0}^{\infty} \frac{K^{i} \varphi\left(m^{i} x_{1}, \ldots, m^{i} x_{n}\right)}{m^{2 i}}<\infty
$$

for all $x_{1}, \ldots, x_{n} \in X$. Then there exists a unique Euler-Lagrange quadratic mapping $Q$ : $X \rightarrow Y$ such that $Q$ satisfies (1.3), that is,

$$
D_{a} Q\left(x_{1}, \ldots, x_{n}\right)=0
$$

for all $x_{1}, \ldots, x_{n} \in X$, and the inequality

$$
\begin{aligned}
\|f(x)-Q(x)\| \leq & \frac{K}{m} \Phi(x, 0, \ldots, 0)+\frac{K}{m^{2}} \Phi\left(a_{1} x, \ldots, a_{n} x\right) \\
& +\frac{K(n-1)(m+1)|n-2 m|\|f(0)\|}{2\left(m^{2}-K\right)}
\end{aligned}
$$

holds for all $x \in X$, where

$$
f(0)=0, \quad \text { if } m<\sqrt{K}, \quad\|f(0)\| \leq \frac{\varphi(0, \ldots, 0)}{\left|m n-\left[1+\left(\begin{array}{c}
n \\
2
\end{array}\right)\right]\right|}, \quad \text { if } m>\sqrt{K} .
$$

The mapping $Q$ is given by

$$
Q(x)=\lim _{k \rightarrow \infty} \frac{f\left(m^{k} x\right)}{m^{2 k}}
$$

for all $x \in X$.

Proof. Substitution of $x_{i}=0(i=1, \ldots, n)$ in the functional inequality (2.4) yields that

$$
\left|m n-\left[1+\left(\begin{array}{l}
n \\
2
\end{array}\right)\right]\right|\|f(0)\| \leq \varphi(0, \ldots, 0) .
$$

Thus we note that if $m<\sqrt{K}$, then $\varphi(0, \ldots, 0)=0$ by the convergence of $\Phi(0, \ldots, 0)$, and so $f(0)=0$. Substituting $x_{1}=x, x_{j}=0(j=2, \ldots, n)$ in the functional inequality $(2.4)$, we obtain

$$
\left\|\sum_{i=1}^{n} f\left(a_{i} x\right)-m f(x)+\left[\left(\begin{array}{c}
n-1 \\
2
\end{array}\right)-m(n-1)\right] f(0)\right\| \leq \varphi(x, 0, \ldots, 0)
$$

or

$$
\left\|\bar{f}^{a}(x, \ldots, x)-f(x)+\left[\frac{1}{m}\left(\begin{array}{c}
n-1 \\
2
\end{array}\right)-(n-1)\right] f(0)\right\| \leq \frac{\varphi(x, 0, \ldots, 0)}{m}
$$


for all $x \in X$. In addition, replacing $x_{i}$ by $a_{i} x$ in (2.4), one gets the inequality

$$
\left\|f(m x)+\left(\begin{array}{l}
n \\
2
\end{array}\right) f(0)-m \sum_{i=1}^{n} f\left(a_{i} x\right)\right\| \leq \varphi\left(a_{1} x, \ldots, a_{n} x\right)
$$

or

$$
\left\|\frac{f(m x)}{m^{2}}+\frac{1}{m^{2}}\left(\begin{array}{l}
n \\
2
\end{array}\right) f(0)-\bar{f}^{a}(x, \ldots, x)\right\| \leq \frac{\varphi\left(a_{1} x, \ldots, a_{n} x\right)}{m^{2}}
$$

for all $x \in X$. From this inequality and (2.12) as well as the triangle inequality, we get the basic inequality

$$
\begin{aligned}
\| \frac{f(m x)}{m^{2}}-f(x) & +\left[\frac{n(n-1)}{2 m^{2}}+\frac{(n-1)(n-2)}{2 m}-(n-1)\right] f(0) \| \\
& \leq \frac{\varphi(x, 0, \ldots, 0)}{m}+\frac{\varphi\left(a_{1} x, \ldots, a_{n} x\right)}{m^{2}}
\end{aligned}
$$

or

$$
\begin{aligned}
& \left\|\frac{f(m x)}{m^{2}}-f(x)\right\| \\
& \quad \leq \varepsilon(x):=\frac{m \varphi(x, 0, \ldots, 0)+\varphi\left(a_{1} x, \ldots, a_{n} x\right)}{m^{2}}+\frac{(n-1)(m+1)|n-2 m|\|f(0)\|}{2 m^{2}}
\end{aligned}
$$

for all $x \in X$.

By induction on $l \in \mathbb{N}$, we prove the general functional inequality

$$
\left\|\frac{f\left(m^{l} x\right)}{m^{2 l}}-f(x)\right\| \leq \frac{K^{l-1} \varepsilon\left(m^{l-1} x\right)}{m^{2(l-1)}}+K \sum_{i=0}^{l-2} \frac{K^{i} \varepsilon\left(m^{i} x\right)}{m^{2 i}}
$$

for all $x \in X$ and all nonnegative integer $l$. In fact, we calculate the inequality

$$
\begin{aligned}
\left\|\frac{f\left(m^{l+1} x\right)}{m^{2(l+1)}}-f(x)\right\| & \leq K\left\|\frac{f\left(m^{l+1} x\right)}{m^{2(l+1)}}-\frac{f(m x)}{m^{2}}\right\|+K\left\|\frac{f(m x)}{m^{2}}-f(x)\right\| \\
& \leq \frac{K}{m^{2}}\left[\frac{K^{l-1} \varepsilon\left(m^{l} x\right)}{m^{2(l-1)}}+K \sum_{i=0}^{l-2} \frac{K^{i} \varepsilon\left(m^{i+1} x\right)}{m^{2 i}}\right]+K \varepsilon(x) \\
& =\frac{K^{l} \varepsilon\left(m^{l} x\right)}{m^{2 l}}+K \sum_{i=0}^{l-1} \frac{K^{i} \varepsilon\left(m^{i} x\right)}{m^{2 i}}
\end{aligned}
$$

for all $x \in X$.

It follows from (2.5) and (2.17) that a sequence $\left\{f_{l}(x)\right\}$ of mappings $f_{l}(x):=f\left(m^{l} x\right) /$ $m^{2 l}$ is Cauchy in the quasi-Banach space $Y$, and it thus converges. Therefore, we see that a mapping $Q: X \rightarrow Y$ defined by

$$
Q(x):=\lim _{l \rightarrow \infty} \frac{f\left(m^{l} x\right)}{m^{2 l}}
$$


6 Journal of Inequalities and Applications

exists for all $x \in X$. Taking the limit $l \rightarrow \infty$ in (2.17), we find that

$$
\begin{aligned}
\|f(x)-Q(x)\| \leq & K \sum_{i=0}^{\infty} \frac{K^{i} \varepsilon\left(m^{i} x\right)}{m^{2 i}} \\
= & \frac{K}{m} \Phi(x, 0, \ldots, 0)+\frac{K}{m^{2}} \Phi\left(a_{1} x, \ldots, a_{n} x\right) \\
& +\frac{K(n-1)(m+1)|n-2 m|\|f(0)\|}{2\left(m^{2}-K\right)}
\end{aligned}
$$

for all $x \in X$. Therefore, the mapping $Q$ near the approximate mapping $f: X \rightarrow Y$ of (1.3) satisfies the inequality (2.7). In addition, it is clear from (2.4) that the following inequality

$$
\frac{1}{m^{2 l}}\left\|D_{a} f\left(m^{l} x_{1}, \ldots, m^{l} x_{n}\right)\right\| \leq \frac{1}{m^{2 l}} \varphi\left(m^{l} x_{1}, \ldots, m^{l} x_{n}\right)
$$

holds for all $x_{1}, \ldots, x_{n} \in X$ and all $l \in \mathbb{N}$. Taking the limit $l \rightarrow \infty$, we see that the mapping $Q$ satisfies the equation $D_{a} Q\left(x_{1}, \ldots, x_{n}\right)=0$, and so $Q$ is Euler-Lagrange quadratic mapping.

Let $\check{Q}: X \rightarrow Y$ be another Euler-Lagrange quadratic mapping satisfying the equation

$$
D_{a} \check{Q}\left(x_{1}, \ldots, x_{n}\right)=0
$$

and the inequality (2.7). To prove the before-mentioned uniqueness, we employ (2.4) so that

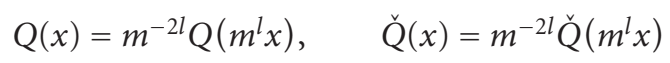

hold for all $x \in X$ and $l \in \mathbb{N}$. Thus from the last equality and inequality (2.7), one proves that

$$
\begin{aligned}
\|Q(x)-\check{Q}(x)\|= & \frac{1}{m^{2 l}}\left\|Q\left(m^{l} x\right)-\check{Q}\left(m^{l} x\right)\right\| \\
\leq & \frac{K}{m^{2 l}}\left(\left\|Q\left(m^{l} x\right)-f\left(m^{l} x\right)\right\|+\left\|f\left(m^{l} x\right)-\grave{Q}\left(m^{l} x\right)\right\|\right) \\
\leq & \frac{2 K^{2}}{m^{2} K^{l}} \sum_{i=0}^{\infty} \frac{m K^{i+l} \varphi\left(m^{i+l} x, 0, \ldots, 0\right)+K^{i+l} \varphi\left(a_{1} m^{i+l} x, \ldots, a_{n} m^{i+l} x\right)}{m^{2(i+l)}} \\
& +\frac{K^{2}(n-1)(m+1) \mid n-2 m\|f(0)\|}{\left(m^{2}-K\right) m^{2 l}}
\end{aligned}
$$

for all $x \in X$ and all $l \in \mathbb{N}$. Therefore, from $l \rightarrow \infty$, one establishes

$$
Q(x)-\check{Q}(x)=0
$$

for all $x \in X$, completing the proof of uniqueness.

Theorem 2.4. Assume that there exists a mapping $\varphi: X^{n} \rightarrow[0, \infty)$ for which a mapping $f: X \rightarrow Y$ satisfies

$$
\left\|D_{a} f\left(x_{1}, \ldots, x_{n}\right)\right\| \leq \psi\left(x_{1}, \ldots, x_{n}\right)
$$


and the series

$$
\Psi\left(x_{1}, \ldots, x_{n}\right):=\sum_{i=1}^{\infty} K^{i} m^{2 i} \psi\left(\frac{x_{1}}{m^{i}}, \ldots, \frac{x_{n}}{m^{i}}\right)<\infty
$$

for all $x_{1}, \ldots, x_{n} \in X$. Then there exists a unique Euler-Lagrange quadratic mapping $Q$ : $X \rightarrow Y$ such that $Q$ satisfies (1.3), that is,

$$
D_{a} Q\left(x_{1}, \ldots, x_{n}\right)=0
$$

for all $x_{1}, \ldots, x_{n} \in X$, and the inequality

$$
\begin{aligned}
\|f(x)-Q(x)\| \leq & \frac{1}{m} \Psi(x, 0, \ldots, 0)+\frac{1}{m^{2}} \Psi\left(a_{1} x, \ldots, a_{n} x\right) \\
& +\frac{K(n-1)(m+1)|n-2 m|\|f(0)\|}{2\left(1-K m^{2}\right)}
\end{aligned}
$$

holds for all $x \in X$, where

$$
f(0)=0, \quad \text { if } m>\frac{1}{\sqrt{K}}, \quad \quad\|f(0)\| \leq \frac{\varphi(0, \ldots, 0)}{\left|m n-\left[1+\left(\begin{array}{c}
n \\
2
\end{array}\right)\right]\right|}, \quad \text { if } m<\frac{1}{\sqrt{K}} .
$$

The mapping $Q$ is given by

$$
Q(x)=\lim _{k \rightarrow \infty} m^{2 k} f\left(\frac{x}{m^{k}}\right)
$$

for all $x \in X$.

Proof. We note that if $m>1 / \sqrt{K}$, then $\psi(0, \ldots, 0)=0$ by the convergence of $\Psi(0, \ldots, 0)$, and so $f(0)=0$. Using the same arguments as those of $(2.12)-(2.17)$, we prove the general functional inequality

$$
\left\|f(x)-m^{2 l} f\left(\frac{x}{m^{l}}\right)\right\| \leq \sum_{i=1}^{l-1} K^{i} m^{2 i} \varepsilon\left(\frac{x}{m^{i}}\right)+K^{l-1} m^{2 l} \varepsilon\left(\frac{x}{m^{l}}\right)
$$

for all $x \in X$ and all nonnegative integer $l>1$, where

$$
\varepsilon(x):=\frac{m \psi(x, 0, \ldots, 0)+\psi\left(a_{1} x, \ldots, a_{n} x\right)}{m^{2}}+\frac{(n-1)(m+1)|n-2 m|\|f(0)\|}{2 m^{2}} .
$$

The rest of the proof goes through by the same way as that of Theorem 2.3.

Corollary 2.5. Let $\mathscr{A}$ be a normed space and $\mathscr{B}$ a Banach space, and let $\theta, p$ be positive real numbers with $p \neq 2$. Assume that a mapping $f: \mathscr{A} \rightarrow \mathscr{B}$ satisfies

$$
\left\|D_{a} f\left(x_{1}, \ldots, x_{n}\right)\right\| \leq \theta\left(\left\|x_{1}\right\|^{p}+\cdots+\left\|x_{n}\right\|^{p}\right)
$$


8 Journal of Inequalities and Applications

for all $x_{1}, \ldots, x_{n} \in \mathscr{A}$. Then there exists a unique Euler-Lagrange quadratic mapping $Q$ : $\mathscr{A} \rightarrow \mathscr{B}$ such that

$$
D_{a} Q\left(x_{1}, \ldots, x_{n}\right)=0
$$

for all $x_{1}, \ldots, x_{n} \in \mathscr{A}$, and

$$
\|f(x)-Q(x)\| \leq \begin{cases}\frac{\theta\|x\|^{p}\left(m+\sum_{i=1}^{n}\left|a_{i}\right|^{p}\right)}{m^{2}-m^{p}} & \text { if } m>1,0<p<2, \\ \frac{\theta\|x\|^{p}\left(m+\sum_{i=1}^{n}\left|a_{i}\right|^{p}\right)}{m^{p}-m^{2}} & \text { or } m<1, p>2, \\ & \text { if } m<1,0<p<2,\end{cases}
$$

for all $x \in \mathscr{A}$.

Remark 2.6. We remark that in Corollary 2.5 the case $p=2$ is not discussed. The EulerLagrange type quadratic functional equation (1.3) is not stable as we will see in the following example with $n=2$. This counterexample is a modification of the example contained in $[26,27]$.

Let us define a mapping $f: \mathbb{R} \rightarrow \mathbb{R}$ by

$$
f(x)=\sum_{n=0}^{\infty} \frac{\varphi\left(2^{n} x\right)}{4^{n}},
$$

where the mapping $\varphi: \mathbb{R} \rightarrow \mathbb{R}$ is given by

$$
\varphi(x)= \begin{cases}1 & \text { if }|x| \geq 1 \\ x^{2} & \text { if }|x|<1\end{cases}
$$

Then the mapping $f$ satisfies the inequality

$$
\begin{aligned}
\mid f\left(a_{1} x_{1}+a_{2} x_{2}\right) & +f\left(a_{2} x_{1}-a_{1} x_{2}\right)-\left(a_{1}^{2}+a_{2}^{2}\right)\left[f\left(x_{1}\right)+f\left(x_{2}\right)\right] \mid \\
& \leq \frac{32}{3}\left(1+a_{1}^{2}+a_{2}^{2}\right)^{2}\left(x^{2}+y^{2}\right)
\end{aligned}
$$

for all $x, y \in \mathbb{R}$, but there exist no Euler-Lagrange quadratic mapping $Q: \mathbb{R} \rightarrow \mathbb{R}$, and a constant $b>0$ such that

$$
|f(x)-Q(x)| \leq b x^{2}
$$

for all $x \in \mathbb{R}$.

In fact, for $x=y=0$ or for $x, y \in \mathbb{R}$ such that $x^{2}+y^{2} \geq 1 / 4\left(1+a_{1}^{2}+a_{2}^{2}\right)$, it is clear that

$$
\begin{aligned}
\mid f\left(a_{1} x_{1}+a_{2} x_{2}\right) & +f\left(a_{2} x_{1}-a_{1} x_{2}\right)-\left(a_{1}^{2}+a_{2}^{2}\right)\left[f\left(x_{1}\right)+f\left(x_{2}\right)\right] \mid \\
& \leq \frac{8}{3}\left(1+a_{1}^{2}+a_{2}^{2}\right) \leq \frac{32}{3}\left(1+a_{1}^{2}+a_{2}^{2}\right)^{2}\left(x^{2}+y^{2}\right)
\end{aligned}
$$


because $|f(x)| \leq 4 / 3$ for all $x \in \mathbb{R}$. Now, we consider the case $0<x^{2}+y^{2}<1 / 4\left(1+a_{1}^{2}+\right.$ $\left.a_{2}^{2}\right)$. Choose a positive integer $k \in \mathbb{N}$ such that

$$
\frac{1}{4^{k+1}\left(1+a_{1}^{2}+a_{2}^{2}\right)} \leq x^{2}+y^{2}<\frac{1}{4^{k}\left(1+a_{1}^{2}+a_{2}^{2}\right)} \text {. }
$$

Then one has $4^{k-1} x^{2}<1 / 4\left|a_{i}\right|^{2}, 4^{k-1} y^{2}<1 / 4\left|a_{i}\right|^{2}$, and so

$$
2^{k-1} x, 2^{k-1} y, 2^{k-1}\left(a_{1} x+a_{2} y\right), 2^{k-1}\left(a_{2} x-a_{1} y\right) \in(-1,1) .
$$

Therefore, we have

$$
2^{n} x, 2^{n} y, 2^{n}\left(a_{1} x+a_{2} y\right), 2^{n}\left(a_{2} x-a_{1} y\right) \in(-1,1)
$$

and hence

$$
\varphi\left(a_{1} x_{1}+a_{2} x_{2}\right)+\varphi\left(a_{2} x_{1}-a_{1} x_{2}\right)-\left(a_{1}^{2}+a_{2}^{2}\right)\left[\varphi\left(x_{1}\right)+\varphi\left(x_{2}\right)\right]=0
$$

for each $n=0,1, \ldots, k-1$. Thus we obtain, using (2.42) and (2.45),

$$
\begin{aligned}
& \left|f\left(a_{1} x_{1}+a_{2} x_{2}\right)+f\left(a_{2} x_{1}-a_{1} x_{2}\right)-\left(a_{1}^{2}+a_{2}^{2}\right)\left[f\left(x_{1}\right)+f\left(x_{2}\right)\right]\right| \\
& \quad \leq \sum_{n=0}^{\infty} \frac{1}{4^{n}}\left|\varphi\left(2^{n}\left(a_{1} x_{1}+a_{2} x_{2}\right)\right)+\varphi\left(2^{n}\left(a_{2} x_{1}-a_{1} x_{2}\right)\right)-\left(a_{1}^{2}+a_{2}^{2}\right)\left[\varphi\left(2^{n} x_{1}\right)+\varphi\left(2^{n} x_{2}\right)\right]\right| \\
& \quad \leq \sum_{n=k}^{\infty} \frac{1}{4^{n}}\left|\varphi\left(2^{n}\left(a_{1} x_{1}+a_{2} x_{2}\right)\right)+\varphi\left(2^{n}\left(a_{2} x_{1}-a_{1} x_{2}\right)\right)-\left(a_{1}^{2}+a_{2}^{2}\right)\left[\varphi\left(2^{n} x_{1}\right)+\varphi\left(2^{n} x_{2}\right)\right]\right| \\
& \quad \leq \sum_{n=k}^{\infty} \frac{2\left(1+a_{1}^{2}+a_{2}^{2}\right)}{4^{n}}=\frac{32\left(1+a_{1}^{2}+a_{2}^{2}\right)}{3 \cdot 4^{k+1}} \leq \frac{32\left(1+a_{1}^{2}+a_{2}^{2}\right)^{2}}{3}\left(x^{2}+y^{2}\right),
\end{aligned}
$$

which yields the inequality (2.39).

Now, assume that there exist an Euler-Lagrange quadratic mapping $Q: \mathbb{R} \rightarrow \mathbb{R}$ and a constant $b>0$ such that

$$
|f(x)-Q(x)| \leq b x^{2}
$$

for all $x \in \mathbb{R}$. Since $|Q(x)| \leq|f(x)|+b x^{2} \leq 4 / 3+b x^{2}$ is locally bounded, the mapping $Q$ is of the form $Q(x)=c x^{2}, x \in \mathbb{R}$ for some constant $c$ [28]. Hence one obtains

$$
|f(x)| \leq(b+|c|) x^{2}
$$

for all $x \in \mathbb{R}$. On the other hand, for $m \in \mathbb{N}$ with $m>b+|c|$ and $x \in\left(0,1 / 2^{m-1}\right)$, we have $2^{n} x \in(0,1)$ for all $n \leq m-1$, and so

$$
f(x)=\sum_{n=0}^{\infty} \frac{\varphi\left(2^{n} x\right)}{4^{n}} \geq \sum_{n=0}^{m-1} \frac{\left(2^{n} x\right)^{2}}{4^{n}}=m x^{2}>(b+|c|) x^{2},
$$

which is a contradiction. 
10 Journal of Inequalities and Applications

Corollary 2.7. Let $\mathscr{A}$ be a normed space, $\mathscr{B}$ a Banach space, and $\theta$, pi positive real numbers such that $p:=\sum_{i=1}^{n} p_{i} \neq 2$. Assume that a mapping $f: \mathscr{A} \rightarrow \mathscr{B}$ satisfies

$$
\left\|D_{a} f\left(x_{1}, \ldots, x_{n}\right)\right\| \leq \theta \prod_{i=1}^{n}\left\|x_{i}\right\|^{p_{i}}
$$

for all $x_{1}, \ldots, x_{n} \in \mathscr{A}$. Then there exists a unique Euler-Lagrange quadratic mapping $Q$ : $\mathscr{A} \rightarrow \mathscr{B}$ such that

$$
D_{a} Q\left(x_{1}, \ldots, x_{n}\right)=0
$$

for all $x_{1}, \ldots, x_{n} \in \mathcal{A}$ and

$$
\|f(x)-Q(x)\| \leq \begin{cases}\frac{\theta\|x\|^{p} \prod_{i=1}^{n}\left|a_{i}\right|^{p_{i}}}{m^{2}-m^{p}} & \text { if } m>1,0<p<2, \\ \frac{\theta\|x\|^{p} \prod_{i=1}^{n}\left|a_{i}\right|^{p_{i}}}{m^{p}-m^{2}} & \text { or } m<1, p>2,0<p<2,0<2\end{cases}
$$

for all $x \in \mathscr{A}$.

In case $n=2$, we have the Hyers-Ulam stability result as a special case of Theorems 2.3 and 2.4 for the Euler-Lagrange type quadratic functional equation (1.2).

Corollary 2.8. Let $\mathscr{A}$ be a linear space, $\mathscr{B}$ a Banach space, and $0 \leq \theta$ a real number. Assume that a mapping $f: \mathscr{A} \rightarrow \mathscr{B}$ satisfies

$$
\left\|D_{a} f\left(x_{1}, \ldots, x_{n}\right)\right\| \leq \theta
$$

for all $x_{1}, \ldots, x_{n} \in \mathscr{A}$. Then there exists a unique Euler-Lagrange quadratic mapping $Q$ : $\mathscr{A} \rightarrow \mathscr{B}$ such that

$$
D_{a} Q\left(x_{1}, \ldots, x_{n}\right)=0
$$

for all $x_{1}, \ldots, x_{n} \in \mathscr{A}$, and the inequality

$$
\|f(x)-Q(x)\| \leq \frac{\theta}{|m-1|}+\frac{\theta(n-1)|n-2 m|\|f(0)\|}{2|m-1|}
$$

for all $x \in \mathscr{A}$.

\section{Stability of (1.3) in Banach modules}

In the last part of this paper, let $B$ be a unital Banach algebra with norm $|\cdot|$, and let ${ }_{B} \mathbb{M}_{1}$ and ${ }_{B} \mathbb{M}_{2}$ be left Banach $B$-modules with norms $\|\cdot\|$ and $\|\cdot\|$, respectively.

As an application of the main Theorem 2.3, we are going to prove the generalized Hyers-Ulam stability problem of the functional equation (1.3) in Banach $B$-modules with the modulus of concavity $K=1$ over a unital Banach algebra. 
Theorem 3.1. Assume that there exists a mapping $\varphi:_{B} \mathbb{M}_{1}^{2} \rightarrow[0, \infty)$ for which a mapping $f:{ }_{B} \mathbb{M}_{1} \rightarrow{ }_{B} \mathbb{M}_{2}$ satisfies

$$
\begin{aligned}
& \left\|D_{a, u} f\left(x_{1}, \ldots, x_{n}\right)\right\| \\
& \quad:=\left\|f\left(\sum_{i=1}^{n} a_{i} u x_{i}\right)+\sum_{1 \leq i<j \leq n} f\left(a_{j} u x_{i}-a_{i} u x_{j}\right)-\left(\sum_{i=1}^{n} a_{i}^{2}\right) u^{2} \sum_{i=1}^{n} f\left(x_{i}\right)\right\| \leq \varphi\left(x_{1}, \ldots, x_{n}\right)
\end{aligned}
$$

for all $x_{1}, \ldots, x_{n} \in{ }_{B} \mathbb{M}_{1}$ and all $u \in B(1):=\{u \in B|| u \mid=1\}$, and the series (2.5) with $K=1$ converges for all $x_{1}, \ldots, x_{n} \in{ }_{B} \mathbb{M}_{1}$. If $f$ is measurable, or for each fixed $x \in{ }_{B} \mathbb{M}_{1}$, the mapping $\mathbb{R} \ni t \rightarrow f(t x) \in{ }_{B} \mathbb{M}_{2}$ is continuous, then there exists a unique Euler-Lagrange quadratic mapping $Q:{ }_{B} \mathbb{M}_{1} \rightarrow{ }_{B} \mathbb{M}_{2}$ such that the following equations

$$
D_{a} Q\left(x_{1}, \ldots, x_{n}\right)=0, \quad Q(b x)=b^{2} Q(x)
$$

and the inequality (2.7) with $K=1$ hold for all $x, x_{1}, \ldots, x_{n} \in{ }_{B} \mathbb{M}_{1}$ and all $b \in B$.

Proof. From (3.1) with $u=1$, it follows by Theorem 2.3 that there exists a unique EulerLagrange quadratic mapping $Q:{ }_{B} \mathbb{M}_{1} \rightarrow{ }_{B} \mathbb{M}_{2}$ such that

$$
D_{a} Q\left(x_{1}, \ldots, x_{n}\right)=0
$$

for all $x_{1}, \ldots, x_{n} \in{ }_{B} \mathbb{M}_{1}$ and the inequality (2.7) with $K=1$ for all $x \in{ }_{B} \mathbb{M}_{1}$.

The mapping $Q$ is given by

$$
Q(x)=\lim _{k \rightarrow \infty} \frac{f\left(m^{k} x\right)}{m^{2 k}}
$$

for all $x \in{ }_{B} \mathbb{M}_{1}$.

Furthermore, suppose that $f$ is measurable, or for each fixed $x \in{ }_{B} \mathbb{M}_{1}$, the mapping $f(t x)$ is continuous with respect to $t \in \mathbb{R}$. Then for any continuous linear functional $L$ defined on ${ }_{B} \mathbb{M}_{2}$, let $\Phi: \mathbb{R} \rightarrow \mathbb{R}$ be given by

$$
\Phi(t):=L[Q(t x)]
$$

for $t \in \mathbb{R}$, where $x$ is fixed. Then $\Phi$ is a quadratic mapping and, moreover, is also measurable since it is the pointwise limit of the sequence

$$
\Phi_{k}(t):=m^{-2 k} L\left[f\left(m^{k} t x\right)\right] .
$$

Hence it has the form $\Phi(t)=t^{2} \Phi(1)$ for all $t \in \mathbb{R}[7]$. Therefore, one obtains that for each fixed $x \in{ }_{B} \mathbb{M}_{1}$ and all $t \in \mathbb{R}$,

$$
L[Q(t x)]=\Phi(t)=t^{2} \Phi(1)=t^{2} L[Q(x)]=L\left[t^{2} Q(x)\right]
$$

which implies the condition

$$
Q(t x)=t^{2} Q(x), \quad \forall x \in{ }_{B} \mathbb{M}_{1}, \forall t \in \mathbb{R} .
$$


12 Journal of Inequalities and Applications

That is, $Q$ is $\mathbb{R}$-quadratic. Replacing $x_{1}, \ldots, x_{n}$ by $m^{k} x_{1}, \ldots, m^{k} x_{n}$ in (3.1), respectively, and dividing it by $m^{2 k}$, we figure out

$$
\frac{\left\|D_{a, u} f\left(m^{k} x_{1}, \ldots, m^{k} x_{n}\right)\right\|}{m^{2 k}} \leq \frac{\varphi\left(m^{k} x_{1}, \ldots, m^{k} x_{n}\right)}{m^{2 k}}
$$

for all $u \in B(1)$ and for all $x_{1}, \ldots, x_{n} \in{ }_{B} \mathbb{M}_{1}$. Taking the limit $k \rightarrow \infty$, one obtains by condition (2.5) that

$$
D_{a, u} Q\left(x_{1}, \ldots, x_{n}\right)=0
$$

for all $x_{1}, \ldots, x_{n} \in{ }_{B} \mathbb{M}_{1}$ and all $u \in B(1)$. Substituting $x_{1}=x, x_{j}=0(j=2, \ldots, n)$ in the functional inequality (3.3), we obtain

$$
\sum_{i=1}^{n} Q\left(a_{i} x\right)-m Q(x)=0
$$

for all $x \in{ }_{B} \mathbb{M}_{1}$. In addition, replacing $x_{i}$ by $a_{i} x$ in (3.10), one gets the inequality

$$
Q(m u x)-m u^{2} \sum_{i=1}^{n} Q\left(a_{i} x\right)=0
$$

or

$$
m Q(u x)-u^{2} \sum_{i=1}^{n} Q\left(a_{i} x\right)=0
$$

for all $x \in{ }_{B} \mathbb{M}_{1}$ and all $u \in B(1)$. Associating the last two equations, we have

$$
Q(u x)-u^{2} Q(x)=0
$$

for all $x \in{ }_{B} \mathbb{M}_{1}$ and all $u \in B(1)$. The last equality is also true for $u=0$ vacuously. Now, for each element $b \in B(b \neq 0)$, we figure out

$$
\begin{aligned}
Q(b x) & =Q\left(|b| \cdot \frac{b}{|b|} x\right)=|b|^{2} \cdot Q\left(\frac{b}{|b|} x\right) \\
& =|b|^{2} \cdot \frac{b^{2}}{|b|^{2}} \cdot Q(x)=b^{2} Q(x)
\end{aligned}
$$

for all $b \in B(b \neq 0)$ and all $x \in{ }_{B} \mathbb{M}_{1}$. Thus the mapping $Q$ satisfies

$$
Q(b x)=b^{2} Q(x)
$$

for all $b \in B$ and for all $x \in{ }_{B} \mathbb{M}_{1}$, as desired. This completes the proof of the theorem.

Alternatively, as an application of the main Theorem 2.4, we obtain the following theorem. 
Theorem 3.2. Assume that a mapping $f:{ }_{B} \mathbb{M}_{1} \rightarrow{ }_{B} \mathbb{M}_{2}$ satisfies

$$
\left\|D_{a, u} f\left(x_{1}, \ldots, x_{n}\right)\right\| \leq \varphi\left(x_{1}, \ldots, x_{n}\right)
$$

for all $x_{1}, \ldots, x_{n} \in{ }_{B} \mathbb{M}_{1}$ and all $u \in B(1)$, and the series (2.27) with $K=1$ converges for all $x_{1}, \ldots, x_{n} \in{ }_{B} \mathbb{M}_{1}$. If $f$ is measurable, or for each fixed $x \in{ }_{B} \mathbb{M}_{1}$, the mapping $\mathbb{R} \ni t \rightarrow f(t x) \in$ ${ }_{B} \mathbb{M}_{2}$ is continuous, then there exists a unique Euler-Lagrange quadratic mapping $Q:{ }_{B} \mathbb{M}_{1} \rightarrow$ ${ }_{B} \mathbb{M}_{2}$ such that the following equations

$$
D_{a} Q\left(x_{1}, \ldots, x_{n}\right)=0, \quad Q(b x)=b^{2} Q(x)
$$

and the inequality (2.29) with $K=1$ hold for all $x, x_{1}, \ldots, x_{n} \in{ }_{B} \mathbb{M}_{1}$ and all $b \in B$.

Since $\mathbb{C}$ is a Banach algebra, the Banach spaces $M_{1}$ and $M_{2}$ are considered as Banach modules over $\mathbb{C}$. Thus we have the following corollary.

Corollary 3.3. Let $\varphi$ be a mapping defined as in Theorem 3.1. Let $M_{1}$ and $M_{2}$ be Banach spaces over the complex field $\mathbb{C}$. Suppose that a mapping $f: M_{1} \rightarrow M_{2}$ satisfies

$$
\left\|D_{a, u} f\left(x_{1}, \ldots, x_{n}\right)\right\| \leq \varphi\left(x_{1}, \ldots, x_{n}\right)
$$

for all $u \in \mathbb{C}(1)$ and all $x_{1}, \ldots, x_{n} \in M_{1}$. If $f$ is measurable or the mapping $\mathbb{R} \ni t \rightarrow f(t x) \in$ $M_{2}$ is continuous for each fixed $x \in M_{1}$, then there exists a unique Euler-Lagrange quadratic mapping $Q: M_{1} \rightarrow M_{2}$ such that the following equations

$$
D_{a} Q\left(x_{1}, \ldots, x_{n}\right)=0, \quad Q(c x)=c^{2} Q(x)
$$

hold for all $x_{1}, \ldots, x_{n} \in M_{1}$ and all $c \in \mathbb{C}$, and the inequality (2.7) with $K=1$ holds for all $x \in M_{1}$.

Theorem 3.4. Assume that there exists a mapping $\varphi:_{B} \mathbb{M}_{1}^{2} \rightarrow[0, \infty)$ for which a mapping $f: \mathbb{M}_{1} \rightarrow{ }_{B} \mathbb{M}_{2}$ satisfies

$$
\left\|u^{2} f\left(\sum_{i=1}^{n} a_{i} x_{i}\right)+\sum_{1 \leq i<j \leq n} u^{2} f\left(a_{j} x_{i}-a_{i} x_{j}\right)-\left(\sum_{i=1}^{n} a_{i}^{2}\right) \sum_{i=1}^{n} f\left(u x_{i}\right)\right\| \leq \varphi\left(x_{1}, \ldots, x_{n}\right)
$$

for all $x_{1}, \ldots, x_{n} \in{ }_{B} \mathbb{M}_{1}$ and all $u \in B(1)$, and the series (2.5) with $K=1$ converges for all $x_{1}, \ldots, x_{n} \in{ }_{B} \mathbb{M}_{1}$. If $f$ is measurable or the mapping $\mathbb{R} \ni t \rightarrow f(t x) \in{ }_{B} \mathbb{M}_{2}$ is continuous for each fixed $x \in{ }_{B} \mathbb{M}_{1}$, then there exists a unique Euler-Lagrange quadratic mapping $Q: \mathbb{M}_{1} \rightarrow{ }_{B} \mathbb{M}_{2}$ such that the following equations

$$
D_{a} Q\left(x_{1}, \ldots, x_{n}\right)=0, \quad Q(b x)=b^{2} Q(x)
$$

and the inequality (2.7) with $K=1$ hold for all $x, x_{1}, \ldots, x_{n} \in{ }_{B} \mathbb{M}_{1}$ and all $b \in B$.

Proof. The proof of this theorem is similar to that of Theorem 3.1.

\section{Acknowledgment}

This study was financially supported by research fund of Chungnam National University in 2007. 


\section{References}

[1] S. M. Ulam, A Collection of Mathematical Problems, Interscience Tracts in Pure and Applied Mathematics, no. 8, Interscience, London, UK, 1960.

[2] P. M. Gruber, "Stability of isometries," Transactions of the American Mathematical Society, vol. 245, pp. 263-277, 1978.

[3] D. X. Zhou, "On a conjecture of Z. Ditzian," Journal of Approximation Theory, vol. 69, no. 2, pp. 167-172, 1992.

[4] D. H. Hyers, "On the stability of the linear functional equation," Proceedings of the National Academy of Sciences, vol. 27, pp. 222-224, 1941.

[5] Th. M. Rassias, "On the stability of the linear mapping in Banach spaces," Proceedings of the American Mathematical Society, vol. 72, no. 2, pp. 297-300, 1978.

[6] P. Găvruţa, "A generalization of the Hyers-Ulam-Rassias stability of approximately additive mappings," Journal of Mathematical Analysis and Applications, vol. 184, no. 3, pp. 431-436, 1994.

[7] D. H. Hyers, G. Isac, and Th. M. Rassias, Stability of Functional Equations in Several Variables, Progress in Nonlinear Differential Equations and Their Applications, 34, Birkhäuser, Boston, Mass, USA, 1998.

[8] D. H. Hyers and Th. M. Rassias, "Approximate homomorphisms," Aequationes Mathematicae, vol. 44, no. 2-3, pp. 125-153, 1992.

[9] Th. M. Rassias, "On the stability of functional equations in Banach spaces," Journal of Mathematical Analysis and Applications, vol. 251, no. 1, pp. 264-284, 2000.

[10] J. M. Rassias, "On the stability of the Euler-Lagrange functional equation," Chinese Journal of Mathematics, vol. 20, no. 2, pp. 185-190, 1992.

[11] J. M. Rassias, "On the stability of the non-linear Euler-Lagrange functional equation in real normed linear spaces," Journal of Mathematical and Physical Sciences, vol. 28, no. 5, pp. 231235, 1994.

[12] J. M. Rassias, "On the stability of the general Euler-Lagrange functional equation," Demonstratio Mathematica, vol. 29, no. 4, pp. 755-766, 1996.

[13] J. M. Rassias, "Solution of the Ulam stability problem for Euler-Lagrange quadratic mappings," Journal of Mathematical Analysis and Applications, vol. 220, no. 2, pp. 613-639, 1998.

[14] J. M. Rassias, "On the stability of the multi-dimensional Euler-Lagrange functional equation," The Journal of the Indian Mathematical Society, vol. 66, no. 1-4, pp. 1-9, 1999.

[15] M. J. Rassias and J. M. Rassias, "On the Ulam stability for Euler-Lagrange type quadratic functional equations," The Australian Journal of Mathematical Analysis and Applications, vol. 2, no. 1, article 11, pp. 1-10, 2005.

[16] K.-W. Jun and H.-M. Kim, "On the stability of Euler-Lagrange type cubic mappings in quasiBanach spaces," Journal of Mathematical Analysis and Applications, vol. 332, no. 2, pp. 13351350, 2007.

[17] Y.-S. Lee and S.-Y. Chung, "Stability of an Euler-Lagrange-Rassias equation in the spaces of generalized functions," to appear in Applied Mathematics Letters.

[18] C. Park and J. M. Park, "Generalized Hyers-Ulam stability of an Euler-Lagrange type additive mapping," Journal of Difference Equations and Applications, vol. 12, no. 12, pp. 1277-1288, 2006.

[19] C.-G. Park, "Hyers-Ulam-Rassias stability of a generalized Euler-Lagrange type additive mapping and isomorphisms between $C^{*}$-algebras," Bulletin of the Belgian Mathematical Society. Simon Stevin, vol. 13, no. 4, pp. 619-632, 2006.

[20] C.-G. Park and J. M. Rassias, "Hyers-Ulam stability of an Euler-Lagrange type additive mapping," International Journal of Applied Mathematics \& Statistics, vol. 7, no. Fe07, pp. 112-125, 2007.

[21] A. Pietrzyk, "Stability of the Euler-Lagrange-Rassias functional equation," Demonstratio Mathematica, vol. 39, no. 3, pp. 523-530, 2006. 
[22] K. Ravi and M. Arunkumar, "On the Ulam-Gavruta-Rassias stability of the orthogonally EulerLagrange type functional equation," International Journal of Applied Mathematics \& Statistics, vol. 7, no. Fe07, pp. 143-156, 2007.

[23] J. M. Rassias, "On the Hyers-Ulam stability problem for quadratic multi-dimensional mappings," Aequationes Mathematicae, vol. 64, no. 1-2, pp. 62-69, 2002.

[24] Y. Benyamini and J. Lindenstrauss, Geometric Nonlinear Functional Analysis. Vol. 1, American Mathematical Society Colloquium Publications, 48, American Mathematical Society, Providence, RI, USA, 2000.

[25] S. Rolewicz, Metric Linear Spaces, PWN-Polish Scientific, Warsaw, Poland, 2nd edition, 1984.

[26] Z. Gajda, "On stability of additive mappings," International Journal of Mathematics and Mathematical Sciences, vol. 14, no. 3, pp. 431-434, 1991.

[27] A. Gilányi, "On the stability of monomial functional equations," Publicationes Mathematicae Debrecen, vol. 56, no. 1-2, pp. 201-212, 2000.

[28] S. Kurepa, "On the quadratic functional," Publications de l'Institut Mathématique de l'Académie Serbe des Sciences et des Arts, vol. 13, pp. 57-72, 1961.

Hark-Mahn Kim: Department of Mathematics, College of Natural Sciences, Chungnam National University, 220 Yuseong-Gu, Daejeon 305-764, South Korea

Email address: hmkim@cnu.ac.kr

John Michael Rassias: Pedagogical Department E. E., National and Capodistrian University of Athens, Section of Mathematics and Informatics, 4 Agamemnonos St., Aghia Paraskevi, Athens 15342, Greece

Email address: jrassias@primedu.uoa.gr

Young-Sun Cho: Department of Mathematics, College of Natural Sciences, Chungnam National University, 220 Yuseong-Gu, Daejeon 305-764, South Korea Email address: yscho@cnu.ac.kr 